\title{
A class of individual-based models
}

\author{
Mirosław Lachowicz \\ Institute of Applied Mathematics and Mechanics \\ University of Warsaw \\ Warsaw, Poland \\ M.Lachowicz@mimuw.edu.pl
}

Received: 9 February 2018, accepted: 12 April 2018, published: 15 April 2018

\begin{abstract}
We discuss a class of mathematical models of biological systems at microscopic level - i.e. at the level of interacting individuals of a population. The class leads to partially integral stochastic semigroups - [25]. We state general conditions guaranteeing the asymptotic stability. In particular under some rather restrictive assumptions we observe that any, even non-factorized, initial probability density tends in the evolution to a factorized equilibrium probability density [16]. We discuss possible applications of the general theory such as redistribution of individuals [10], thermal denaturation of DNA [7], and tendon healing process $-[11]$.
\end{abstract}

Keywords-Individual-based models; Markov jump processes; Integro-differential equations, Stochastic semigroups, Stability.

\section{MICROSCOPIC SCALE}

In the present paper we review the general class of individual-based models in Biology developed in Ref. [15] — see also [3], [14], [16] and references therein. We show that the class corresponds to the partially integral stochastic semigroups and under some more restrictive assumptions leads to the stability result. We discuss possible applica- tions of the general theory such as redistribution of individuals - [10], thermal denaturation of DNA [7], and tendon healing process - [11].

We consider the general equation that defines the evolution of a number $N$ of individuals of biological populations — cf. Refs. [3], [14], [15] and references therein. Each individual $n(n \in$ $\{1, \ldots, N\}$ ) is characterized by its inner (microscopic) state

$$
u_{n} \in \mathbb{U}
$$

where $\mathbb{U}$ is a Borel set in $\mathbb{R}^{d}, d \in\{1,2,3, \ldots\}$. The variable $u_{n}$ related to the individual $n$ may have various meanings: it may be any vector parameter that characterize an individual biological state of any of the individuals. In particular it may also contain an information of a subpopulation to which the individuals belongs (a discrete component) - see [3], [14], [15].

In the general setting $(\mathbb{U}, \mathfrak{B}, \mu)$ is a $\sigma$-finite measure space. In some applications $\mathbb{U}$ is a product of a discrete set and a Lebesgue-measurable subset (e.g. a closed bounded interval) in the discretecontinuous picture or a discrete set in the discretediscrete case and the measure $\mu$ is a product of the

Copyright: (C) 2018 Lachowicz et al. This article is distributed under the terms of the Creative Commons Attribution License (CC BY 4.0), which permits unrestricted use, distribution, and reproduction in any medium, provided the original author and source are credited.

Citation: Mirosław Lachowicz, A class of individual-based models, Biomath 7 (2018), 1804127, 
counting measure and the counting measure (in the discrete-discrete picture) or the Lebesgue measure (in the discrete-continuous picture).

The $n_{1}$-individual changes its state at random times. We consider the possibility of the following stochastic changes

- without interactions,

- due to interaction with the $n_{2}$-individual, $n_{2} \in\{1, \ldots, N\}, n_{2} \neq n_{1}$,

- due to interaction with the $n_{2}$ and $n_{3}$ individuals,

- ...

- due to interaction with $n_{2}, n_{3}, \ldots, n_{M}$ individuals, where $M$ is an integer $2 \leq M \leq N$.

Consider interactions of the given individual with $m-1$ individuals, $m=1, \ldots, M$.

Assumption 1. The rate of interaction between the individual with state $u_{n_{1}}$ and the individuals with states $u_{n_{2}}, \ldots, u_{n_{m}}$ is given by the measurable function $a^{[m]}=a^{[m]}\left(u_{n_{1}}, \ldots, u_{n_{m}}\right)$, such that

$$
0 \leq a^{[m]}\left(u_{n_{1}}, u_{n_{2}}, \ldots, u_{n_{m}}\right) \leq a_{+}^{[m]},
$$

for all $u_{n_{1}}, u_{n_{2}}, \ldots, u_{n_{m}} \in \mathbb{U}$, where $a_{+}^{[m]}<\infty$ is a constant.

Assumption 2. The transition into state $v$ of an $n_{1}$-individual with state $u_{n_{1}}$, due to the interaction with individuals of $n_{2}, \ldots, n_{m}$ with states $u_{n_{2}}, \ldots, u_{n_{m}}$, respectively, is described by the measurable function $A^{[m]}=A^{[m]}\left(v ; u_{n_{1}}, \ldots, u_{n_{m}}\right) \geq$ 0 , where

$$
\int_{\mathbb{U}} A^{[m]}\left(v ; u_{n_{1}}, u_{n_{2}}, \ldots, u_{n_{m}}\right) \mathrm{d} \mu(v)=1,
$$

for all $u_{n_{1}}, u_{n_{2}}, \ldots, u_{n_{m}} \in \mathbb{U}$.

The stochastic model (at the microscopic level) is determined by the functions $a^{[m]}$ and $A^{[m]}$.

$\begin{gathered}L_{1}^{(N)} \text { is the } \quad \text { space } \\ \text { with the norm }\|f\|_{L_{1}^{(N)}}\end{gathered}$
$\int_{\mathbb{U}^{N}}\left|f\left(u_{1}, \ldots, u_{N}\right)\right| \mathrm{d} \mu\left(u_{1}\right) \ldots \mathrm{d} \mu\left(u_{N}\right)$. If $N=1$

we simply write $L_{1}$.

Given $N, M$, and $a^{[m]}, A^{[m]}$, for $m=$ $1, \ldots, M$, we consider the stochastic system that is defined by the Markov jump process of $N$ individuals through the following generator $\Lambda$ acting on densities

$$
\Lambda=\Lambda^{+}-\Lambda^{-}=\sum_{m=1}^{M}\left(\Lambda^{[m]+}-\Lambda^{[m]-}\right),
$$

$\Lambda^{[m]+} f\left(t, u_{1}, u_{2}, \ldots, u_{N}\right)=$

$\mathfrak{c}_{N, m} \sum_{\substack{1 \leq n_{1}, \ldots, n_{m} \leq N \\ n_{i} \neq n_{j} \forall i \neq j}} \int_{\mathbb{U}} A^{[m]}\left(u_{n_{1}} ; v, u_{n_{2}}, \ldots, u_{n_{m}}\right) \times$ $a^{[m]}\left(v, u_{n_{2}}, \ldots, u_{n_{m}}\right) \times$

$f\left(t, u_{1}, \ldots, u_{n_{1}-1}, v, u_{n_{1}+1}, \ldots, u_{N}\right) \mathrm{d} \mu(v)$,

$$
\begin{aligned}
& \Lambda^{[m]-} f\left(t, u_{1}, u_{2}, \ldots, u_{N}\right)= \\
& \mathfrak{c}_{N, m} \sum_{\substack{1 \leq n_{1}, \ldots, n_{m} \leq N \\
n_{i} \neq n_{j} \forall i \neq j}} a^{[m]}\left(u_{n_{1}}, u_{n_{2}}, \ldots, u_{n_{m}}\right) \times \\
& f\left(t, u_{1}, \ldots, u_{N}\right),
\end{aligned}
$$

on $\mathbb{U}^{N}$, where $\mathfrak{c}_{N, m}=\frac{1}{(m-1) !\left(\begin{array}{c}N \\ m-1\end{array}\right)}$ are normalizing constants.

Assume that the system is initially distributed according to $F \in L_{1}^{(N)}$ and time evolution is described by the following (linear) equation - the modified Liouville equation,

$$
\frac{\partial}{\partial t} f=\Lambda f, ;\left.\quad f\right|_{t=0}=F
$$

with the initial data

$$
\left.f\right|_{t=0}=F .
$$

We refer here to the Liouville equation in the sense of particle dynamics: Eq. (3) plays a similar role as the Liouville equation in kinetic theory see Ref. [6] (cf. also [18]). The generator $\Lambda$ is the difference between the gain term and loss terms $\Lambda=\Lambda^{+}-\Lambda^{-}$, where

- the gain term $\Lambda^{+}$is a sum of terms describing the changes from state $v$ of $n_{1}$-individual into $u_{n_{1}}$ due to the interaction with $n_{2}, \ldots, n_{m}$ individuals with states $u_{n_{2}}, \ldots, u_{n_{m}}$, respectively for $2 \leq m \leq M$ and the term $(m=1)$ describing the direct changes of state $v$ of $n_{1}-$ individual into $u_{n_{1}}$ without interactions;

- the loss term $\Lambda^{-}$is a sum of terms describing the changes from state $u_{n_{1}}$ of $n_{1}$-individual into another state due to the interaction with 
$n_{2}, \ldots, n_{m}$ individuals with states $u_{2}, \ldots, u_{m}$, respectively for $2 \leq m \leq M$ or without interactions for $m=1$.

It is easy to see that under Assumptions $1-2$ the operator $\Lambda$ is a bounded in $L_{1}^{(N)}$. Thus the Cauchy Problem has the unique solution

$$
f(t)=\exp (t \Lambda) F
$$

in $L_{1}^{(N)}$ for all $t \geq 0$. The solution is nonnegative for nonnegative initial data and the $L_{1}^{(N)}$-norm is preserved for any $t>0$. Therefore $\{\exp (t \Lambda)\}_{t \geq 0}$ defines a continuous (linear) semigroup of Markov operators that is a stochastic semigroup in the sense of Ref. [21]. Actually we may note that here we have even a group.

In Ref. [15] (see also [14], [3] and the references therein) the limit $N \rightarrow \infty$ was studied. Under suitable assumption Eq. (3) results in a nonlinear kinetic equation referred to the corresponding mesoscopic level. Moreover in various cases macroscopic limits can be obtain.

In the next section (Section II) we show that $\{\exp (t \Lambda)\}_{t \geq 0}$ is a partially integral stochastic semigroup (see Refs. [26], [25]) and, under some additional assumptions, leads to a stability result. In Section III we review some possible applications.

\section{AsYMPtotic BEHAVIOUR}

In order to formulate the time asymptotic result we can refer to the notion of the partially integral stochastic semigroups — see Refs. [26], [25]) and the Lower Function Theorem by Lasota and Yorke - see [22] (Theorem 2; cf. also Corollary IV.16 in Ref. [28]).

Using similar strategy as in Refs. [16] we prove a more general result that may be related to a general class of microscopic systems in the form given by Eq. (5) under reasonably general Assumptions 1 and 2 .

Lemma II.1. Let Assumptions 1 and 2 be satisfied. Assume moreover that $a^{[m]}$ is non-zero, for some $m \in\{1, \ldots, M\}$. Then $\{\exp (t \Lambda)\}_{t \geq 0}$ is a partially integral stochastic semigroup.
Proof: Let $\Gamma$ be the operator given by

$$
\Gamma F=\Lambda F+a^{+} F,
$$

for $F \in L_{1}^{(N)}$, where $a^{+}=\max _{m=1, \ldots, M} a^{[m]}$. Then

$$
\Gamma F \geq \Lambda^{+} F \geq \max \{0, \Lambda F\}
$$

and

$$
\exp (t \Lambda) F=\exp \left(-a^{+} t\right) \exp (t \Gamma) F,
$$

for any probability density $F$ on $\mathbb{U}^{N}$.

By Eqs. (6) and (7), for any probability density $F$ on $\mathbb{U}^{N}$, we have

$$
\begin{aligned}
& \exp (t \Lambda) F\left(u_{1}, \ldots, u_{N}\right) \\
& \geq \frac{t^{N}}{N !} \exp \left(-a^{+} t\right)\left(\Lambda^{+}\right)^{N} F\left(u_{1}, \ldots, u_{N}\right) \\
& \geq \mathfrak{c}_{N, m}(t) \int_{\mathbb{U}^{N}} k^{[m}\left(u_{1}, \ldots, u_{N}, v_{1}, \ldots, v_{N}\right) \\
& \quad \times F\left(v_{1}, \ldots, v_{N}\right) \mathrm{d} \mu\left(v_{1}\right) \ldots \mathrm{d} \mu\left(v_{N}\right),
\end{aligned}
$$

where $\mathfrak{c}_{N, m}(t)$ is a constant that depends on $m, n$ and $t>0$ and $k^{[m]}$ is a complicated function that depends on $A^{[m]}$ and $a^{[m]}$,

$$
\begin{aligned}
k^{[m]} & \left(u_{1}, \ldots, u_{N}, v_{1}, \ldots, v_{N}\right)= \\
= & A^{[m]}\left(u_{1} ; v_{1}, u_{2}, \ldots u_{m}\right) a^{[m]}\left(v_{1}, u_{2}, \ldots u_{m}\right) \\
& \times A^{[m]}\left(u_{2} ; v_{2}, u_{3}, \ldots u_{m+1}\right) a^{[m]}\left(v_{2}, u_{3}, \ldots u_{m+1}\right) \\
& \times \ldots A^{[m]}\left(u_{N} ; v_{N}, v_{1}, \ldots v_{m-1}\right) \\
& \times a^{[m]}\left(v_{N}, v_{1}, \ldots v_{m-1}\right) .
\end{aligned}
$$

Thus (see [26]) $\{\exp (t \Lambda)\}_{t \geq 0}$ is a partially integral stochastic semigroup.

We note that the above result does not need any additional assumption. The class of partially integral stochastic semigroups is particularly important (see [25], [26], [28]) in the analysis of asymptotic behaviour of stochastic semigroups. To state the asymptotic stability we need however a stronger assumption

Theorem II.2. Let Assumptions 1 and 2 be satisfied. Additionally, for some $m \in\{1, \ldots, M\}$ we assume that $a^{[m]}$ is nonzero, and there exists a measurable nonnegative function $h$ on $\mathbb{U}$ such that

$$
\int_{\mathbb{U}} h(u) \mathrm{d} \mu(u)>0,
$$


and

$$
A^{[m]}\left(v, u_{1}, \ldots, u_{m}\right) a^{[m]}\left(u_{1}, \ldots, u_{m}\right) \geq h(v),
$$

for any $v, u_{1}, u_{2}, \ldots, u_{m} \in \mathbb{U}$. Then the stochastic semigroup $\{\exp (t \Lambda)\}_{t \geq 0}$ is asymptotically stable.

Proof: As in the proof of Lemma II.1, for any probability density $F$ on $\mathbb{U}^{N}$, we have

$$
\begin{aligned}
& \exp (t \Lambda) F\left(u_{1}, \ldots, u_{N}\right) \\
& \geq \quad \frac{t^{N}}{N !} \exp \left(-a^{+} t\right)\left(\Lambda^{+}\right)^{N} F\left(u_{1}, \ldots, u_{N}\right) \\
& \geq \mathfrak{c}_{N, m}(t) \prod_{i=1}^{N} h\left(u_{i}\right) \\
& \quad \times \int_{\mathbb{U}^{N}} F\left(v_{1}, \ldots, v_{N}\right) \mathrm{d} \mu\left(v_{1}\right) \ldots, \mathrm{d} \mu\left(v_{N}\right) \\
& \quad=\mathfrak{c}_{N, m}(t) \prod_{i=1}^{N} h\left(u_{i}\right),
\end{aligned}
$$

where $\mathfrak{c}_{N, m}(t)$ is a constant that depends on $m, n$ and $t>0$. Let $t_{0}>0$ be fixed, e.g. $t_{0}=1$. We have

$$
\exp (t \Lambda) F=\exp \left(t_{0} \Lambda\right) \exp \left(\left(t-t_{0}\right) \Lambda\right) F
$$

By Eq. (11) we obtain

$$
\begin{aligned}
& \exp (t \Lambda) F\left(u_{1}, \ldots, u_{N}\right) \\
& \geq \mathfrak{c}_{N, m}\left(t_{0}\right) \prod_{i=1}^{N} h\left(u_{i}\right)\left\|\exp \left(\left(t-t_{0}\right) \Lambda\right) F\right\|_{L_{1}^{(N)}},
\end{aligned}
$$

for $t>t_{0}$. Keeping in mind that $\exp \left(\left(t-t_{0}\right) \Lambda\right) F$ is a probability density, for each $t>t_{0}$ we conclude

$$
\exp (t \Lambda) F\left(u_{1}, \ldots, u_{N}\right) \geq \ell\left(u_{1}, \ldots, u_{N}\right),
$$

where $\ell\left(u_{1}, \ldots, u_{N}\right)=\mathfrak{c}_{N, m}\left(t_{0}\right) \prod_{i=1}^{N} h\left(u_{i}\right)$ depends on $N$ but does not depend on $t$ and $F$. By Assumptions 11 and 2 it follows that $\ell \in L_{1}^{(N)}$. Moreover by Eq. (9)

$$
\int_{\mathbb{U}^{N}} \ell\left(u_{1}, \ldots, u_{N}\right) \mathrm{d} \mu\left(u_{1}\right) \ldots \mathrm{d} \mu\left(u_{N}\right)>0,
$$

and $\ell$ is a lower function in the sense of Lasota and Yorke [22]. In fact the following condition holds

$$
\lim _{t \rightarrow \infty}\left\|(\exp (t \Lambda) F-\ell)^{-}\right\|_{L_{1}^{(N)}}=0
$$

for every probability density $F$, where $X^{-}=0$ if $X \geq 0$ and $X^{-}=-X$ if $X<0$. Thus by the lower function theorem of Lasota and Yorke the semigroup $\{\exp (t \Lambda)\}_{t \geq 0}$ is asymptotically stable.

As a by-product of Theorem II.2 we obtain the uniqueness of an equilibrium (stationary) solution corresponding to Eq. (3). The identification of possible equilibrium solutions is an essential step in studying macroscopic limits corresponding to the microscopic models - see [3].

In a particular case referred to a microscopic system in Ref. [16], under some (rather strong) assumption, it is shown that any, even nonfactorized, initial probability density tends in the evolution to a factorized equilibrium probability density. Such a situation one can refer to as asymptotic annihilation of initial correlations in the system. On the other hand it was also shown that if the mentioned assumptions are not satisfied - a number of equilibrium states could be large and no annihilation is observed.

The possible relationships between micro-, meso- and macro- scales were discussed in Ref. [3] (see also references therein) - Chapter 8 and in particular Subsection 8.3.3.

\section{ApPLICATIONS}

There are many possible applications of the general theory presented in section I. The stochastic systems that corresponds at the macroscopic level to standard logistic growth were considered in Ref. [16]. The parameter $u_{n}, n \in\{1,2, \ldots, N\}$, describing the microscopic (individual) state of $n$-individual, may be related to its activity (cf. Refs. [4], [3]). The parameter may also describe dominance [13] or social state (c.f. Refs. [1], [5], [9]). The references mentioned above refer to the mesoscopic (kinetic) description whereas Ref. [16] - to microscopic (individual-based) one.

Usually the importance of the microscopic approach may be particularly visible in a case when the number of interacting entities of the system is not huge which is typical for biological systems. In such cases the kinetic (mesoscopic) description not always may be properly justified. 


\section{A. Redistribution of individuals}

An application of the general model at the microscopic level of the redistribution of individuals in a closed domain featuring, as an example, an elevator — see [10]. The modeling bases on experiments performed in order to elucidate the interactions between pedestrians - see [12] and the references in [10]. In these experiments the inflow of persons into a spatially restricted area, e.g. an elevator, was studied, featuring the process inverse to evacuation. In Ref. [10] an $n$-th individual, $n=1, \ldots, N$, is characterized by its position $u_{n}$. The model and analysis presented in [10] have a preliminary nature and still have to be developed.

\section{B. Thermal denaturation of DNA}

In Ref. [7] some aspects of deoxyribonucleic acid DNA thermal denaturation process (cf. [23], [24], [2]) were considered. Ref. [7] is a continuing the idea of [8] where a preliminary mesoscopic (kinetic) model was discussed. In Ref. [7] a new, more adequate, model that takes into account at the individual (microscopic) level the time evolution of the probability distribution of the state of all hydrogen bonds. Two types of bonds (with two and three hydrogen bonds) and the direct dependence on the temperature are included in the model. The base pairs A-T, C-G are numbered by the discrete variable $n \in\{1,2, \ldots, N\}$, and the continuous variable $u \in[0, \infty[$ representing the stretching of the distance between the two connected base is used. The variable $u$ is called stretching parameter. Every base pair ('bond') $n$ is then characterized by the variables $u_{n}$. The discrete variable belongs to one of two subsets of bonds: $\mathbb{J}_{2}$ - two hydrogen bonds connecting $\mathrm{A}$ and $\mathrm{T}$ and $\mathbb{J}_{3}-$ three hydrogen bonds connecting $\mathrm{C}$ and $\mathrm{G}$

$$
\mathbb{J}_{2} \cup \mathbb{J}_{3}=\mathbb{J}, \quad \mathbb{J}_{2} \cap \mathbb{J}_{3}=\emptyset .
$$

According to the biological knowledge it is assumed that the three hydrogen bonds are more resistant to heating than the two hydrogen bonds. The probability densities $f=f\left(t, u_{1}, \ldots, u_{N}\right)$ that describe the distribution of the variable $u_{1}, \ldots, u_{N}$ at all bonds is considered.

\section{Tendon healing process}

In Ref. [11] a kinetic model of collagen remodeling occurring in latter stage of tendon healing process was proposed and studied. The model is an integro-differential equation describing the alignment of collagen fibers in a finite time. An important feature of tendon structure is the collagen fibers orientation. In the healthy tendon they are aligned. The result of the tendon injury is a disturbance of the parallel structure. The healing process consists in the reconstruction of parallel structure. Scars that may be formed during the healing process cause no proper alignment of collagen fibers. One of the most important indicators of the success of the treatment of tendon injury is the degree of alignment of collagen fibers.

The model in [11] refers to the function $g(t, x, v)$ that describes a statistical state of collagen, i.e. the probability density $g=g(t, x, v)$ to find a collagen fiber at the instant of time $t>0$ at point $x \in \mathbb{D}$ and with orientation $v \in \mathbb{V}$, where $\mathbb{D}, \mathbb{V}$ are domains in $\mathbb{R}^{d}$. Thus the model has a mesoscopic nature.

We consider the following equation

$$
\begin{gathered}
\frac{\partial}{\partial t} g(t, x, v)=\int_{\mathbb{V}} \int_{\mathbb{D}}\left(T_{g}(y, v ; x, w) g(t, x, w)\right. \\
\left.-g(t, x, v) T_{g}(y, w ; x, v)\right) \mathrm{d} y \mathrm{~d} w
\end{gathered}
$$

where $T_{g}(y, v ; x, w)$ describes the transition probability from the orientation $w \in \mathbb{V}$ at $x \in \mathbb{D}$ to the orientation $v \in \mathbb{V}$ at $x$ caused by an adaptation to the orientation at $y \in \mathbb{D}$. The model bases on a proper choice of the function $T_{f}$ that in general may depend on both collagen distribution $g$ and tenocytes density $c$. In Ref. [11] a simplified case of constant (uniform) tenocytes density was considered.

A realistic definition is

$$
T_{g}(y, v ; x, w)=\beta(y, v ; x, w) g^{\gamma}(t, y, v),
$$

where $\gamma>0$ describes the strength of influence of collagen fibers from neighborhood on collagen fiber in considered point. The bigger is the $\gamma$ the stronger the influence is. That choice leads to the 
following general class of equations

$$
\begin{aligned}
& \frac{\partial}{\partial t} g(t, x, v)= \\
& \int_{\mathbb{D}} \int_{\mathbb{V}}\left(\beta(y, v ; x, w) g^{\gamma}(t, y, v) g(t, x, w)\right. \\
& \left.-g(t, x, v) \beta(y, w ; x, v) g^{\gamma}(t, y, w)\right) \mathrm{d} y \mathrm{~d} w .
\end{aligned}
$$

The function $\beta(y, v, x, w)$ is related to the interaction between the collagen fiber with orientation $w$ at point $x$ with collagen fiber with orientation $v$ located at point $y$ and describes the transition from orientation $w$ to orientation $v$.

In Ref. [11] we show that the solutions may exist globally in time or may blow-up in a finite time depending on initial data. The latter behavior is related to the healing of injury without the scar formation in a finite time: a full alignment of collagen fibers occurs. The approach of [11] may be related to the mesoscopic scale.

One may however propose a stochastic individually based (microscopic) model following the idea stated in Section I. The number $N$ may be then related to the number of collagen fibers that are taken into account in the modeling process. The system is described in terms of a Markov jump process and the related linear evolution equations as in Section I. The equation describes the evolution of probability densities, with microscopic representation of the system of $N$ interacting agents.

We consider the interactions of a given agent with $\gamma$ agents. The system is initially distributed according to the probability density $F \in L_{1}^{(N)}$.

The time evolution is described by Eq. (3) where $\Lambda$ is the generator that takes the form

$$
\begin{aligned}
& \Lambda f\left(t, x_{1}, v_{1}, \ldots, x_{N}, u_{N}\right)= \\
& \quad \mathfrak{c}_{N, \gamma} \sum_{\substack{1 \leq n_{1}, \ldots, n_{\gamma+1} \leq N \\
n_{i} \neq n_{j} \forall i \neq j}}\left(\int_{\mathbb{D} \times \mathbb{V}}\right. \\
& A\left(x_{n_{1}}, v_{n_{1}} ; y, w, x_{n_{2}}, v_{n_{2}}, \ldots, v_{n_{\gamma+1}}, v_{n_{\gamma+1}}\right) \\
& \times a\left(y, w, x_{n_{2}}, v_{n_{2}}, \ldots, x_{n_{\gamma+1}}, v_{n_{\gamma+1}}\right) \\
& \times f\left(t, x_{1}, v_{1}, \ldots, x_{n_{1}-1}, v_{n_{1}-1}, y, w\right. \\
& \left.x_{n_{1}+1}, v_{n_{1}+1}, \ldots, x_{N}, v_{N}\right) \mathrm{d} y \mathrm{~d} w \\
& -a\left(x_{n_{1}}, v_{n_{1}}, \ldots, x_{n_{\gamma+1}}, v_{n_{\gamma+1}}\right) \\
& \left.f\left(t, x_{1}, v_{1}, \ldots, x_{N}, v_{N}\right)\right) .
\end{aligned}
$$

In the limit $N \rightarrow \infty$, the (linear) modified Liouville equation (3) yields, [15], [3], a nonlinear integro-differential equation that can be related to the mesoscopic description

$$
\begin{aligned}
& \frac{\partial}{\partial t} f(t, u)=\mathcal{G}[f](t, u)-f(t, u) \mathcal{L}[f](t, u), \\
& u=(x, v) \in \mathbb{D} \times \mathbb{V}
\end{aligned}
$$

where $\mathcal{G}[f]$ is the gain term, given by

$$
\begin{aligned}
& \mathfrak{G}[f](t, x, v)=\sum_{\{\}} \int_{(\mathbb{D} \times \mathbb{V})^{\gamma+1}} \\
& A\left(x, v ; y, w,\left\{x_{2}, v_{2}, \ldots, x_{\gamma+1}, u_{\gamma+1}\right\}\right) \\
& \times a\left(y, w,\left\{x_{2}, u_{2}, \ldots, x_{\gamma+1}, u_{\gamma+1}\right\}\right) \\
& \times f(t, y, w) f\left(t, x_{2}, v_{2}\right) \ldots f\left(t, x_{\gamma+1}, v_{\gamma+1}\right) \\
& \mathrm{d} y \mathrm{~d} w \mathrm{~d} x_{2}, \mathrm{~d} v_{2}, \ldots \mathrm{d} x_{\gamma+1}, \mathrm{~d} v_{\gamma+1},
\end{aligned}
$$

and $f \mathcal{L}[f]$ is the loss term, defined as

$$
\begin{aligned}
& \mathfrak{L}[f](t, x, v)=\sum_{\{\}} \int_{(\mathbb{D} \times \mathbb{V})^{\gamma}} \\
& a\left(x, v,\left\{x_{2}, v_{2}, \ldots, x_{\gamma+1}, v_{\gamma+1}\right\}\right) \\
& \times f\left(t, x_{2}, v_{2}\right) \ldots f\left(t, x_{\gamma+1}, v_{\gamma+1}\right) \\
& \times \mathrm{d} x_{2} \mathrm{~d} v_{2} \ldots \mathrm{d} x_{\gamma+1} \mathrm{~d} v_{\gamma+1},
\end{aligned}
$$

and $\sum_{\{\}}$means the sum over all permutation of variables within \{\} .

It is easy to see that the global (in time) existence and uniqueness of solutions $f=f(t)$ to Eq. (17) in $L_{1}^{(1)}$ follows.

One may now state the theorem (cf. [15], [3]) that defines the links between the solutions to Eq. (3) and to Eq. (17) or, in other words, that defines the transition from the microscopic level to the mesoscopic level.

The mathematical properties of Eq. (17) are different than those of Eq. (16). The possible rich behavior of solutions of Eq. (16), see [11], [17], leading to blow-ups in a finite of time are not possible in the case of solutions of Eq. (17). On the other hand in some limit (approximating "delta-function") the solutions of Eq. (16) may be approximated by the solutions of Eq. (17). This defines the relationship between a stochastic, individually-based (microscopic) description and Eq. (16). 
A formal series with respect to $\gamma=1,2, \ldots$, results in the following nonlinear kinetic (mesoscopic) equation alternative to Eq. 16

$$
\begin{aligned}
& \frac{\partial}{\partial t} g(t, x, v)= \\
& \int_{\mathbb{D}} \int_{\mathbb{V}}\left(\beta(y, v ; x, w) \frac{g(t, y, v)}{1-g(t, y, v)} g(t, x, w)\right. \\
& \left.-g(t, x, v) \beta(y, w ; x, v) \frac{g(t, y, w)}{1-g(t, y, w)}\right) \mathrm{d} y \mathrm{~d} w .
\end{aligned}
$$

At present, the mathematical theory of Eq. (18) is missing.

\section{CONCLusions}

In Section [1] we review the general class of microscopic models that are able to describe interactions between individuals of a biological population. The class refers to the stochastic semigroups. In Section $\amalg$ we show the methods that leads to the asymptotic stability under some rather restrictive assumptions. On the other hand the asymptotic behaviour in the general case is still an open problem. The important technical tool could be the fact that the semigroup is partially integral (without any particular additional assumption). This may be treated as a preliminary step towards the description of macroscopic ("hydrodynamic") limits that seems to be essential part of the program of giving full description on various scales starting from microscopic, then mesoscopic and finally macroscopic.

In a very simple case considered in [16] the macroscopic equation was obtain from the mesoscopic equation by the averaging with respect to microscopic variable. In the general case it is far from being solved. Therefore we may believe that the methods of the present paper can indicate the possible further research.

In Section III we review some important applications. They show that the general framework is suitable to describe various systems in which the interactions between individuals are essential.

We point out some new equations that result in various limits that can be interesting for further mathematical studies.

\section{ACKNOWLEDGMENT}

The author was supported by the National Science Centre Poland Grant 2017/25/B/ST1/00051.

\section{REFERENCES}

[1] G. Ajmone Marsan, N. Bellomo and M. Egidi, Towards a mathematical theory of complex socioeconomical systems by functional subsystems representation, Kinetic Rel. Models, 1:2 (2008), 249-278; DOI:10.3934/krm.2008.1.249.

[2] A.E. Allahverdyan, Z.H.S. Gevorkian, C.-K. Hu, and Th.M. Nieuwenhuizeni, How absorption influences DNA denaturation, Phys. Rev. E, 79 (2000), 4988-4991.

[3] J. Banasiak and M. Lachowicz, Methods of small parameter in mathematical biology, Birkhäuser/Springer, Cham, Basel 2014.

[4] N. Bellomo, N.K. Li, and Ph.K. Maini, On the foundations of cancer modelling: Selected topics, speculations and perspectives, Math. Models Methods Appl. Sci., 18 (2008), 593-646.

[5] N. Bellomo, M.A. Herrero, and A. Tosin, On the dynamics of social conflicts looking for the Black Swan, Kinetic Relat. Models, 6 (2013), 459-479; 10.3934/krm.2013.6.459.

[6] C. Cercignani, R. Illner, and M. Pulvirenti, The Mathematical Theory of Dilute Gases, Springer, New York, Berlin 1994.

[7] M. Debowski, M. Lachowicz, and Z. Szymańska, Microscopic description of DNA thermal denaturation, to appear.

[8] M. Dolfin and M. Lachowicz, Modeling DNA thermal denaturation at the mesoscopic level, Discrete Contin. Dyn. Syst. Ser. B, 19, 8 (2014), 2469-2482.

[9] M. Dolfin and M. Lachowicz, Modeling altruism and selfishness in welfare dynamics: The role of nonlinear interactions, Math. Models Methods Appl. Sci., 24: 12 (2014), 2361-2381; DOI:10.1142/S0218202514500237.

[10] M. Dolfin, M. Lachowicz, and A. Schadschneider, A microscopic model of redistribution of individuals inside an 'elevator', In Modern Problems in Applied Analysis, P. Drygaś and S. Rogosin (Eds.), Bikhäuser, Basel (2018), 77-86; DOI: 10.1007/978-3-319-72640-3.

[11] G. Dudziuk, M. Lachowicz, H. Leszczyński, and Z. Szymańska, A simple model of collagen remodeling, to appear.

[12] T. Ezaki, K. Ohtsuka, M. Chraibi, M. Boltes, D. Yanagisawa, A. Seyfried, A. Schadschneider, and K. Nishinari, Inflow process of pedestrians to a confined space, Collective Dynamics 1(A:4), 2016, 1-18.

[13] E. Jäger and L. Segel, On the distribution of dominance in a population of interacting anonymous organisms, SIAM J. Appl. Math. 52 (1992), 1442-1468.

[14] M. Lachowicz, Microscopic, mesoscopic and macroscopic descriptions of complex systems, Prob. Engin. Mech., 26:1 (2011), 54-60; DOI: 10.1016/j.probengmech.2010.06.007 
[15] M. Lachowicz, Individually-based Markov processes modeling nonlinear systems in mathematical biology, Nonlinear Analysis Real World Appl., 12 (2011), 23962407;

DOI: 10.1016/j.nonrwa.2011.02.014

[16] M. Lachowicz, A class of microscopic individual models corresponding to the macroscopic logistic growth, Math. Methods Appl. Sci., 2017, on-line, DOI: 10.1002/mma.4680

[17] M. Lachowicz, H. Leszczyński, and M. Parisot, Blowup and global existence for a kinetic equation of swarm formation, Math. Models Methods Appl. Sci., 27 (2017), 1153-1175.

[18] M. Lachowicz and M. Pulvirenti, A stochastic particle system modeling the Euler equation, Arch. Rational Mech. Anal. 109 (1), 1990, 81-93.

[19] M. Lachowicz and T. Ryabukha, Equilibrium solutions for microscopic stochastic systems in population dynamics, Math. Biosci. Engineering, 10:3 (2013), 777-786; DOI: $10.3934 / \mathrm{mbe} .2013 .10 .777$

[20] M. Lachowicz and D. Wrzosek, Nonlocal bilinear equations. Equilibrium solutions and diffusive limit, Math. Models Methods Appl. Sci., 11 (2001), 13751390;
DOI: $10.1142 / \mathrm{S} 0218202501001380$

[21] A. Lasota, M.C. Mackey, Chaos, Fractals, and Noise, Springer, New York 1994.

[22] A. Lasota and J.A. Yorke, Exact dynamical systems and the Frobenius-Perron operator, Trans. Amer. Math. Society, 273 (1982), 375-384.

[23] M. Peyrard, Nonlinear dynamics and statistical physics of DNA, Nonlinearity, 17 (2004), R1-R40.

[24] M. Peyrard, S. Cuesta-López, and G. James, Modelling DNA at the mesoscale: a challenge for nonlinear science?, Nonlinearity, 21 (2008), T91-T100.

[25] K. Pichór and R. Rudnicki, Continuous Markov semigroups and stability of transport equations, J. Math. Analysis Appl., 249, 2000, 668-685, DOI: 10.1006/jmaa.2000.6968

[26] R. Rudnicki, Markov operators: Applications to diffusion processes and population dynamics, Appl. Math. (Warsaw), 27, 1 (2000), 67-79.

[27] R. Rudnicki, Models of population dynamics and genetics, in From Genetics to Mathematics, Eds.: M. Lachowicz and J. Miękisz, World Sci., New Jersey 2009, 103-147.

[28] R. Rudnicki, Models and Methods in Mathematical Biology, In Polish, IMPAN, Warszawa 2014. 